\title{
Outcomes of Metallic Stents for Malignant Ureteral Obstruction
}

\author{
Zachariah G. Goldsmith,* Agnes J. Wang,* Lionel L. Bañez,† Michael E. Lipkin,‡ \\ Michael N. Ferrandino,§ Glenn M. Preminger* and Brant A. Inman*, , \\ From the Division of Urologic Surgery, Duke University Medical Center, Durham, North Carolina
}

Purpose: Malignant ureteral obstruction often necessitates chronic urinary diversion and is associated with high rates of failure with traditional ureteral stents. We evaluated the outcomes of a metallic stent placed for malignant ureteral obstruction and determined the impact of risk factors previously associated with increased failure rates of traditional stents.

Materials and Methods: Patients undergoing placement of the metallic Resonance ${ }^{\circledR}$ stent for malignant ureteral obstruction at an academic referral center were identified retrospectively. Stent failure was defined as unplanned stent exchange or nephrostomy tube placement for signs or symptoms of recurrent ureteral obstruction (recurrent hydroureteronephrosis or increasing creatinine). Predictors of time to stent failure were assessed using Cox regression.

Results: A total of 37 stents were placed in 25 patients with malignant ureteral obstruction. Of these stents $12(35 \%)$ were identified to fail. Progressive hydroureteronephrosis and increasing creatinine were the most common signs of stent failure. Three failed stents had migrated distally and no stents required removal for recurrent infection. Patients with evidence of prostate cancer invading the bladder at stent placement were found to have a significantly increased risk of failure (HR $6.50,95 \%$ CI $1.45-29.20, p=0.015$ ). Notably symptomatic subcapsular hematomas were identified in 3 patients after metallic stent placement.

Conclusions: Failure rates with a metallic stent are similar to those historically observed with traditional polyurethane based stents in malignant ureteral obstruction. The invasion of prostate cancer in the bladder significantly increases the risk of failure. Patients should be counseled and observed for subcapsular hematoma formation with this device.

Key Words: stents, ureteral obstruction

MaLignant ureteral obstruction can result from a variety of malignancies via extrinsic compression of the ureter from a primary or metastatic mass effect. $^{1-3}$ The prognosis for these patients is universally poor, with multiple studies reporting overall survival of less than 1 year after intervention for MUO. ${ }^{4-6}$ Ureteral stenting may be indicated to minimize pain or to maximize renal function, especially if further chemotherapy is planned. ${ }^{3}$
Unfortunately failure rates of $35 \%$ to $45 \%$ have been reported for traditional polyurethane ureteral stents in MUO. $^{7-9}$

A metallic coiled Double-J® ureteral stent manufactured from a nickelcobalt-chromium-molybdenum alloy was recently developed (Resonance $\AA$, Cook Medical, Bloomington, Indiana). This stent has a reported dwell time of up to 12 months and has been used clinically since $2006 .{ }^{10}$ Failure rates have

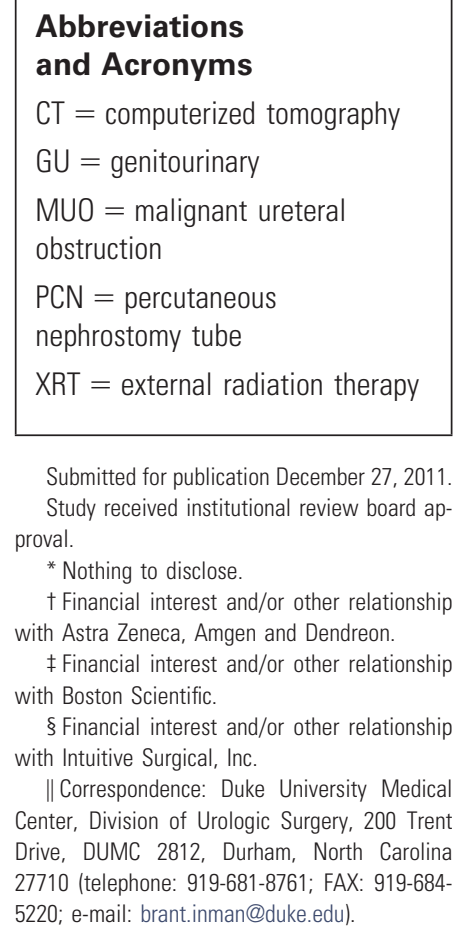

Submitted for publication December 27, 2011 Study received institutional review board ap-

${ }^{*}$ Nothing to disclose.

$\dagger$ Financial interest and/or other relationship with Astra Zeneca, Amgen and Dendreon.

‡ Financial interest and/or other relationship with Boston Scientific.

$\S$ Financial interest and/or other relationship with Intuitive Surgical, Inc.

|| Correspondence: Duke University Medical Center, Division of Urologic Surgery, 200 Trent Drive, DUMC 2812, Durham, North Carolina 27710 (telephone: 919-681-8761; FAX: 919-6845220; e-mail: brant.inman@duke.edu). proval

Editor's Note: This article is the fifth of 5 published in this issue for which category $1 \mathrm{CME}$ credits can be earned. Instructions for obtaining credits are given with the questions on pages 1054 and 1055.

www.jurology.com 
varied from $7 \%$ to $66 \%$ in a small number of retrospective series. ${ }^{11-15}$ Of note, although no prior studies have focused exclusively on patients with MUO, subset analyses revealed higher failure rates with MUO in multiple series. ${ }^{11,14,15}$

Given the indefinite need for urinary diversion in MUO, these patients would benefit a great deal from more durable ureteral stents. Therefore, we reviewed the outcomes of this stent when placed specifically for MUO. In addition, given this novel stent material and design, we hypothesized that risk factors for failure which have been identified for traditional stents, including the presence of malignant bladder invasion, ${ }^{9}$ would not impact the outcome of this metallic device.

\section{MATERIALS AND METHODS}

Data from all patients undergoing placement of the metallic Resonance ureteral stent at Duke University Medical Center were retrospectively obtained following institutional review board approval. Inclusion criteria were biopsy proven malignancy and hydroureteronephrosis consistent with MUO on CT, ultrasound, magnetic resonance, diuretic renography or retrograde urography. The study interval was September 2010 through July 2011. Covariates assessed included age, gender, type of underlying malignancy, laterality, level of obstruction, stent length, prior ureteral stent or $\mathrm{PCN}$ placement, prior radiation treatment, urine cultures, serum creatinine, presence of ileal conduit, and presence of visible tumor seen invading the bladder at the time of stent placement.

All stents were placed in a retrograde fashion with fluoroscopic guidance. No stents were placed anterograde and no stent placement attempts were unsuccessful. Ureteral balloon dilation was not required for any case. Nine different surgeons placed the stents. All stents had a $6 \mathrm{Fr}$ diameter and length ranged from 20 to $28 \mathrm{~cm}$. Following retrograde urography the supplied outer sheath with inner cannula was placed coaxially over a guidewire advanced to the level of the renal pelvis under fluoroscopy. The guidewire and inner cannula were then removed, and the stent was advanced through the sheath, using the cannula as a pusher, until a proximal curl was observed fluoroscopically in the renal pelvis. The sheath was then withdrawn over the cannula such that the distal stent curl was observed fluoroscopically in the bladder. Final fluoroscopy images were obtained to confirm proper stent position before concluding the procedure. Postoperative studies included serum creatinine with abdominal plain films, CT or ultrasound at intervals determined by the treating physician.

Stent failure was defined as unanticipated stent exchange or nephrostomy tube placement for signs or symptoms of recurrent ureteral obstruction (recurrent hydroureteronephrosis or increasing creatinine). Increased creatinine without concomitant hydroureteronephrosis, suggestive of intrinsic or prerenal disease, was not considered stent failure. Median followup duration was calculated with a reverse Kaplan-Meier estimator. Predictors of time to stent failure were assessed using univariate Cox regression. Three patients were identified with no followup data after the initial stent insertion and were censored from this time to event analysis. Univariate analyses were used for predictive calculations as the low number of events precluded accurate multivariate regression modeling. All analyses were conducted using STATA ${ }^{\circledR}$ v.11 with a 2 -sided alpha of 0.05 .

\section{RESULTS}

A total of 37 stents were placed in 25 patients (18 men, 7 women). Twenty-one (56\%) were placed for GU malignancies, and 16 were placed for other malignancies including gastrointestinal (19\%, 7 of 37), gynecologic (16\%, 6 of 37), lymphoma (3\%, 1 of 37$)$ and other (5\%, 2 of 37). Seven patients underwent bilateral stent placement. Stents were placed as conversion from traditional stents $(49 \%, 18$ of 37$)$, as the initial intervention for MUO (19\%, 7 of 37), as salvage interventions in patients in whom traditional stents had failed (19\%, 7 of 37) or as replacements in those with metallic stent failure $(14 \%, 5$ of $37)$. There were $21(57 \%)$ patients with prior external radiation therapy to the abdomen or pelvis. Bladder invasion visualized on cystoscopy was present in 4 patients (11\%), all with prostate cancer. Three patients died with a stent in place. An additional 3 patients were lost to followup (not seen at our institution in the last 12 months) and, thus, were censored from our outcome analyses.

After a median followup of 13 weeks 12 stents (35\%) had failed. Progressive hydroureteronephrosis (58\%, 7 of 12) and increasing creatinine (50\%, 6 of 12 ) were the most common signs of stent failure (not mutually exclusive). Three failed stents (25\%) had migrated distally. None of the 6 stents placed for proximal ureteral obstruction failed. No stents required removal for recurrent infection. Median time to failure was 14 weeks from placement. The majority of stent failures (75\%, 9 of 12) were identified by serum creatinine or imaging obtained during routine oncologic surveillance. Stent failures were managed by placing an alternative ureteral stent (17\%, 2 of 12) or nephrostomy tube (17\%, 2 of 12$)$. Two cases $(17 \%)$ of stent migration with a mild increase in creatinine were managed with observation and $1(8 \%)$ migrated stent associated with significant hydronephrosis was managed with metallic stent replacement. Five failed metallic stents were managed with removal and replacement with a second metallic stent, and none of these replacements went on to fail during the study period. Of the 9 patients without discomfort at the time of stent failure, hydronephrosis or renal failure stabilized in 4 , improved in 3 , worsened in 1 or was not assessed in 1 after intervention. One patient (3\%, 1 of 37$)$ progressed to a scheduled metallic stent exchange during the study period. 
We next investigated possible predictors of clinical outcome (see table). Because of the novel stent material and design, we hypothesized that risk factors for failure identified for traditional stents, including the presence of malignant bladder invasion, ${ }^{9}$ distal level of obstruction ${ }^{8}$ and increased serum creatinine, ${ }^{1,8}$ would not impact the outcome of this metallic device. Notably Cox regression analysis revealed that patients with prostate cancer invading the bladder at cystoscopy had a significantly increased risk of failure (HR 6.50, 95\% CI 1.45-29.20, $\mathrm{p}=0.015$ ). Stent failure occurred in 1 patient with an ileal conduit in concordance with previously reported higher rates of metallic stent failure in this population. ${ }^{16} \mathrm{XRT}$, which has been shown to predict failure with metallic stents in 1 previous study, ${ }^{14}$ was not associated with a significant difference in outcome in this series $(\mathrm{p}=0.244)$.

Notably, subcapsular hematomas developed in 3 patients after metallic stent placement. All 3 patients had stents placed by a single surgeon, who placed the majority of stents in this series. The affected patients presented with similar findings of persistent flank pain, mild anemia (mean decrease in hemoglobin $1.8 \mathrm{gm}$ ) and absence of leukocytosis or fever. A representative CT demonstrated a subcapsular hematoma of the right kidney, with the proximal stent coil in the expected position in the renal pelvis (fig. 1). Mean time to presentation for this complication was 14 days after stent placement (range 3 to 20). Each of these cases was managed conservatively and none required transfusion or intervention. All 3 cases improved on serial imaging.

\section{DISCUSSION}

Given the poor overall survival of patients with MUO,${ }^{4-6}$ the goals of intervention are to provide durable urinary diversion in properly selected patients, with a minimum number of repeat interventions and minimal impact on the quality of remaining life. Diversion with PCN appeared to be associated with lower rates of failure compared to internal ureteral stents in a single retrospective study of 148 patients with MUO. ${ }^{17}$ However, a survey of urolo-

Risk factors for stent failure in MUO

\begin{tabular}{lrrr}
\hline & Hazard Ratio $(95 \% \mathrm{Cl})$ & $\mathrm{p}$ Value \\
\hline Bladder invasion & 6.50 & $(1.45-29.20)$ & 0.015 \\
Prior XRT & 2.20 & $(0.59-8.24)$ & 0.244 \\
Prior stent & 3.72 & $(0.47-29.23)$ & 0.212 \\
Prior PCN & 1.81 & $(0.49-6.73)$ & 0.378 \\
Age & 1.02 & $(0.97-1.07)$ & 0.432 \\
GU vs nonGU Ca & 1.35 & $(0.40-4.52)$ & 0.629 \\
Stent length & 0.42 & $(0.53-3.25)$ & 0.403 \\
Ileal conduit & 13.94 & $(0.26-154.02)$ & 0.032 \\
\hline
\end{tabular}

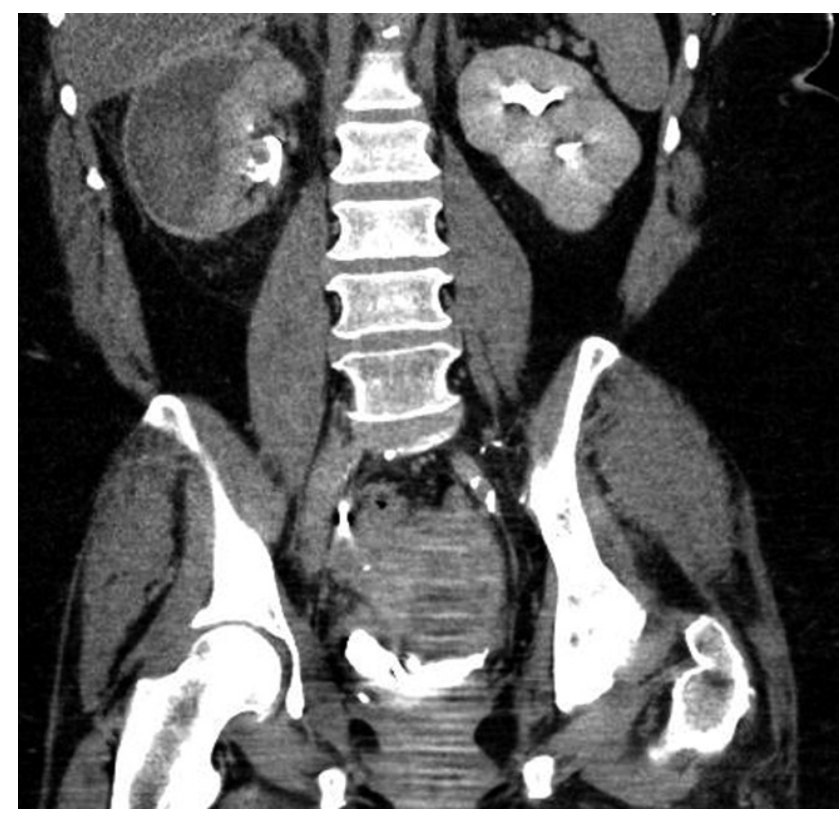

Figure 1. CT obtained 14 days after metallic stent placement reveals right subcapsular hematoma.

gists and medical oncologists indicated a preference to manage most cases of MUO with internal stents, citing concerns about PCN dislodgement and infection. ${ }^{18}$

Failure rates for traditional polyurethane based stents in MUO range from approximately $35 \%$ to $45 \% .^{7-9}$ A failure rate of $43 \%$ (20 of 46 stents) for extrinsic ureteral obstruction was first reported by Docimo and Dewolf. ${ }^{7}$ Similar failure rates of $42 \%$ (58 of 138$)^{1}$ and $36 \%(56 \text { of } 157)^{9}$ were reported in 2 larger retrospective series. In a prospective study of stent placement in 92 patients with intrinsic obstruction or malignant external obstruction, the MUO subset had significantly higher rates of failure (44\% vs $27 \%, \mathrm{p}=0.001$ ). ${ }^{8}$ Taken together, these findings underscore the need for novel therapeutic approaches for MUO.

Since its first application, ${ }^{10}$ several recent studies have characterized the outcomes of the metallic stent retrospectively. ${ }^{11-15}$ Overall failure rates in cohorts including MUO and nonMUO cases have ranged widely from $7 \%$ to $66 \% .^{11-15}$ Of note, although no prior studies have focused exclusively on patients with MUO, this subset of patients was found to have higher failure rates in 3 previous series. ${ }^{11,14,15}$ Other series have demonstrated equivalent $^{13}$ or even improved outcomes ${ }^{12}$ in patients with MUO.

To our knowledge this is the first series to specifically analyze the outcomes of this device in MUO. For this analysis our definition of failure was similar to comparable studies, ${ }^{11-15}$ as unplanned stent exchange or nephrostomy tube placement for signs or 
symptoms of recurrent ureteral obstruction (recurrent hydroureteronephrosis or increasing creatinine). In applying this standard to our cohort of 37 stents placed for MUO, a failure rate of $35 \%$ was identified. The observation that median time to failure (14 weeks) was longer than the median followup for subjects in whom stents did not fail (13 weeks) suggests that the true failure rate for MUO may be higher if confirmed with studies involving longer followup.

Malignant invasion into the bladder noted on cystoscopy has been identified as a risk factor for failure with traditional polyurethane stents. ${ }^{9}$ This suggests that external compression or malignant stent ingrowth may predispose standard stents to higher failure rates. In vitro studies have demonstrated that the metallic stent can withstand a greater magnitude of externally applied force compared to polyurethane stents. ${ }^{19}$ This stent was shown to maintain patent anterograde flow in a porcine model of external ureteral compression in vivo. ${ }^{20}$ Therefore, we hypothesized that malignant bladder invasion would not increase the risk of clinical failure with this stent. In this context our finding that prostate cancer invading into the bladder does indeed significantly increase the risk of metallic stent failure in MUO is highly significant. The prior observation that this stent functions primarily by mediating extraluminal flow $^{20}$ suggests that an obliterative process at the level of the ureteral orifice or trigone, rather than malignant stent ingrowth, may serve as the mechanism of failure in these patients. Our findings suggest that patients with malignant bladder invasion are best treated with external PCN drainage.

XRT was associated with higher failure rates with metallic stents in 1 prior series. ${ }^{14}$ In contrast, another study reported no difference in stent outcomes between XRT and nonXRT cases. ${ }^{11}$ XRT did not appear to predict outcome in our series. Some authors have suggested impaired ureteral peristalsis secondary to fibrosis as a mechanism contributing to stent failure after XRT. ${ }^{14}$ This phenomenon may be dependent on timing and dose of XRT, neither of which were measured in ours or prior studies.

We have provided the first description of subcapsular hematoma formation following the placement of these stents in 3 patients. These patients did not have diagnosed coagulopathies and only 1 was receiving systemic anticoagulation perioperatively (325 mg aspirin daily). A Sensor ${ }^{\mathrm{TM}}$ PTFE-Nitinol Guidewire was used to place 2 of these stents, and an Amplatz Super Stiff ${ }^{\mathrm{TM}}$ guidewire was used in 1 patient. Neither guidewire perforation nor contrast extravasation was noted at stent placement. We believe that hematoma formation may be related to excessive length of the inner cannula relative to the outer sheath in the supplied introducer system. A radiopaque marker is located at the distal end of this outer sheath. Thus, when the radiopaque marker of this introducer system is advanced to the renal pelvis, it is likely that the inner cannula projects further into the renal tissue. To avoid this complication we now routinely cut off the distal tip of the inner cannula to the same level as the open-ended sheath before advancing the introducer system over a guidewire (fig. 2).

Despite the high cost of the stent itself, a recent analysis in a series of 13 patients with chronic benign obstruction reported an overall decrease in health care cost with this device compared to traditional stents during a 1-year period due to the decreased number of operative procedures required to exchange the stents in the Resonance group. ${ }^{21} \mathrm{How}_{-}$ ever, the $35 \%$ failure rate requiring additional interventions in our series suggests that decreased operative cost savings may not be maintained in patients with MUO. In addition, patients in whom a subcapsular hematoma developed required an average of 4 followup imaging tests per patient, with an estimated cost of $\$ 1,500$ (2011 Medicare payments in North Carolina were $\$ 209$ for noncontrast CT, $\$ 415$ for CT with/without contrast, $\$ 174$ for lasix renal scan). Although failed stents remained patent for a median of 14 weeks before failure, taken together, our findings call into question the overall cost-effectiveness of this device for MUO.

This study has the limitations inherent to all retrospective, single institution studies. In addition, due to the novelty of this device, the high rate of death in MUO and the number of patients lost to

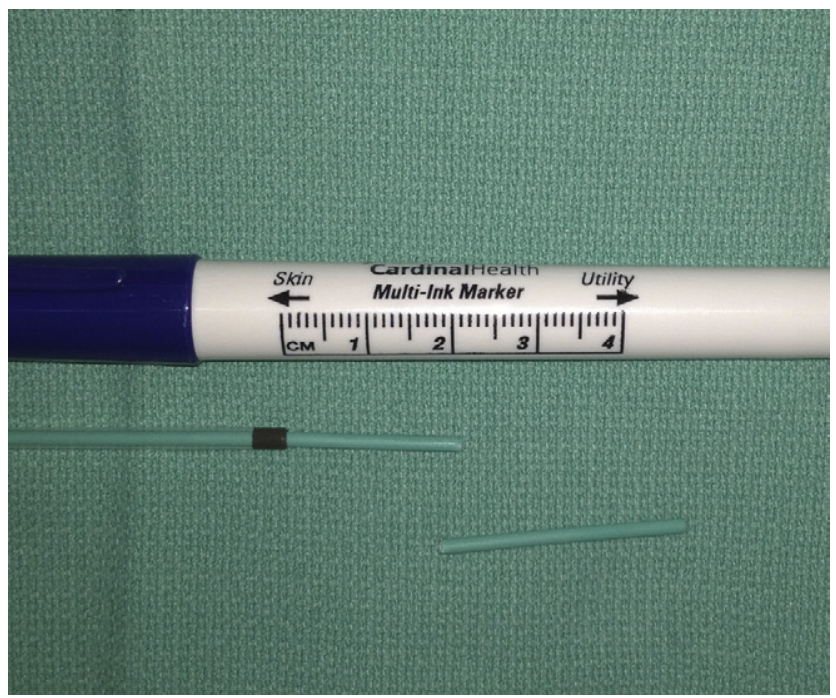

Figure 2. Metallic stent introducer system may be modified by cutting distal tip closer to radiopaque marker. 
followup at our center, the sample size of our series was small and underpowered for multivariate modeling. However, this series of 37 stents is similar in size to most series recently published on this device, ${ }^{11,12,14,15}$ and the first to focus exclusively on MUO. In addition, it would be interesting to assess the impact of the metallic stent on health related quality of life in patients with MUO in a prospective trial setting.

\section{CONCLUSIONS}

The present study identified a $35 \%$ rate of failure in patients with MUO treated with a metallic ureteral stent. This outcome is comparable to the $35 \%$ to $45 \%$ failure rates historically observed with traditional polyurethane based stents for MUO. ${ }^{7-9}$ Patients with prostate cancer with malignant bladder invasion on cystoscopy are at significantly higher risk for failure. These observations warrant confirmation in randomized prospective clinical trials. Patients electing treatment with this device should be appropriately counseled and observed for postoperative subcapsular hematoma formation, which may be related to placement technique with the supplied introducer system. When selecting stents, urologists should consider that the reported dwell time of this metallic stent may not be achievable in patients with MUO.

\section{REFERENCES}

1. Chung SY, Stein RJ, Landsittel D et al: 15-Year experience with the management of extrinsic ureteral obstruction with indwelling ureteral stents. J Urol 2004; 172: 592.

2. Kouba E, Wallen EM and Pruthi RS: Management of ureteral obstruction due to advanced malignancy: optimizing therapeutic and palliative outcomes. J Urol 2008; 180: 444.

3. Allen DJ, Longhorn SE, Philp T et al: Percutaneous urinary drainage and ureteric stenting in malignant disease. Clin Oncol (R Coll Radiol) 2010; 22: 733

4. Radecka $E$, Magnusson $M$ and Magnusson A: Survival time and period of catheterization in patients treated with percutaneous nephrostomy for urinary obstruction due to malignancy. Acta Radiol 2006; 47: 328.

5. Wong LM, Cleeve LK, Milner AD et al: Malignant ureteral obstruction: outcomes after intervention. Have things changed? J Urol 2007; 178: 178.

6. Ishioka J, Kageyama Y, Inoue M et al: Prognostic model for predicting survival after palliative urinary diversion for ureteral obstruction: analysis of 140 cases. J Urol 2008; 180: 618.

7. Docimo SG and Dewolf WC: High failure rate of indwelling ureteral stents in patients with extrin- sic obstruction: experience at 2 institutions. J Urol 1989; 142: 277.

8. Yossepowitch 0, Lifshitz DA and Dekel Y: Predicting the success of retrograde stenting for managing ureteral obstruction. J Urol 2001; 166: 1746 .

9. Ganatra AM and Loughlin KR: The management of malignant ureteral obstruction treated with ureteral stents. J Urol 2005; 174: 2125.

10. Borin JF, Melamud 0 and Clayman RV: Initial experience with full-length metal stent to relieve malignant ureteral obstruction. J Endourol 2006; 20: 300.

11. Nagele U, Kuczyk MA, Horstmann M et al: Initial clinical experience with full-length metal ureteral stents for obstructive ureteral stenosis. World J Urol 2008; 26: 257.

12. Liatsikos $E$, Kallidonis $P$, Kyriazis I et al: Ureteral obstruction: is the full metallic double-pigtail stent the way to go? Eur Urol 2010; 57: 480.

13. Modi AP, Ritch CR, Arend D et al: Multicenter experience with metallic ureteral stents for malignant and chronic benign ureteral obstruction. J Endourol 2010; 24: 1189.

14. Wang HJ, Lee TY, Luo HL et al: Application of resonance metallic stents for ureteral obstruction. BJU Int 2011; 108: 428.
15. Benson AD, Taylor ER and Schwartz BF: Metal ureteral stent for benign and malignant ureteral obstruction. J Urol 2011; 185: 2217.

16. Garg T, Guralnick ML, Langenstroer P et al: Resonance metallic ureteral stents do not successfully treat ureteroenteric strictures. J Endourol 2009; 23: 1199.

17. Ku JH, Lee SW, Jeon HG et al: Percutaneous nephrostomy versus indwelling ureteral stents in the management of extrinsic ureteral obstruction in advanced malignancies: are there differences? Urology 2004; 64: 895.

18. Hyams ES and Shah 0: Malignant extrinsic ureteral obstruction: a survey of urologists and medical oncologists regarding treatment patterns and preferences. Urology 2008; 72: 51.

19. Christman MS, L'esperance J0, Choe CH et al: Analysis of ureteral stent compression force and its role in malignant obstruction. J Urol 2009; 181: 392.

20. Blaschko SD, Deane LA, Krebs A et al: In-vivo evaluation of flow characteristics of novel metal ureteral stent. J Endourol 2007; 21: 780.

21. López-Huertas HL, Polcari AJ, Acosta-Miranda A et al: Metallic ureteral stents: a cost-effective method of managing benign upper tract obstruction. J Endourol 2010; 24: 483. 